\title{
Pairing of off-center polarons due to dipole-dipole interaction: possible clue to the high-temperature superconductivity
}

\author{
M. Borissov and M. Georgiev \\ Institute of Solid Physics, Bulgarian Academy of Sciences, Sofia, Bulgaria \\ Z. Phys. B - Condensed Matter 70, 413-420 (1988)
}

We acknowledge having made the following misprint on filling in the mathematical formulae in the manuscript:

The external (hydrogen-atom like) part of the $2 p$-type wave function in (15) on page 415 has erroneously been written down in the $2 s$-form. The correct mathematical expression for the radial part of $\psi_{2 p}(r)$ (out) should, therefore, read $B_{2 p} a_{2 p} r \exp \left(-a_{2 p} r\right)$.

Fortunately, all the pertaining calculations that follow from there on have been made using the correct $2 p$-wavefunction.

M. Borissov, M. Georgiev Institute of Solid Physics

Bulgarian Academy of Sciences

Boul. Lenin 72

BG-1784 Solia

Bulgaria 